\title{
SENSING IMMOBILIZED MOLECULES OF STREPTAVIDIN ON A SILICON SURFACE BY MALDI-TOF MASS SPECTROMETRY AND FLUORESCENCE MICROSCOPY
}

\author{
EYLEEN ARAYA*1, MARCELO J. KOGAN², ALEIX G. GUELL ${ }^{3}$, CARLOS A. ESCOBAR ${ }^{1}$, FAUSTO SANZ*3,4 \\ ${ }^{1}$ Departamento de Ciencias Químicas, Facultad de Ciencias Exactas, Universidad Andres Bello. Av. Republica 275, Santiago, Chile. \\ ${ }^{2}$ Departamento de Farmacología y Toxicología, Facultad de Ciencias Químicas, Universidad de Chile. Sergio Livingstone 1007, Santiago, Chile. \\ Advanced Center for Chronic Diseases (ACCDiS) \\ ${ }^{3} I B E C$, Institute of Bioengineer of Catalonia, Baldiri Reixac, 1008028 Barcelona, Spain. \\ ${ }^{4}$ Departament de Química Fisica, Universitat de Barcelona, Martí i Franqués 1, 08028 Barcelona, Spain.
}

\begin{abstract}
A hydrogen-terminated $\mathrm{Si}$ (111) surface was modified to form an aminoterminated monolayer for immobilization of streptavidin. Cleavage of an $\mathrm{N}-(\omega-$ undecylenyl)-phthalimide covered surface using hidrazine yields an amino group-modified surface, which serves as a substrate for the attachment of biotin and subsequently streptavidin. We used surface analytical techniques to characterize the surface and to control the course of functionalization before the immobilization of streptavidin. To confirm the presence of the streptavidin Texas red on the surface two powerful techniques available in a standard biochemical laboratory are used, Fluorescence Microscopy and MALDI-TOF that allow us to detect and determine the immobilized streptavidin. This work provides an avenue for the development of devices in which the exquisite binding specificity of biomolecular recognition is directly coupled to a biosensor. In addition, we have demonstrated that MALDI-TOF and fluorescence microscopy are useful techniques for the characterization of silicon functionalized surfaces.
\end{abstract}

\section{INTRODUCTION}

Biosensors have attracted considerable interest in recent years since the monitoring of a specific substance is now central in many applications ranging from clinical analysis to environmental control and for monitoring many industrial processes. A biosensor employs a biological or biochemical detector, which can range from single proteins and enzymes up to whole cells and microorganisms ${ }^{1}$.

Atomic force microscopy (AFM) provides a relatively new approach to the study of specific biointeractions $\mathrm{s}^{1-4}$. In the last decade, using AFM, several research groups observed that microcantilevers can transduce a number of different kinds of signals, e.g. mass, temperature, heat, electromagnetic field and stress into a mechanical deformation: either a deflection or a change in the resonance frequency, with a resolution some orders of magnitude higher than that achievable with microelectronic devices. For example, a small cantilever can be used as a sensitive mass detector by monitoring the resonant frequency of the cantilever, which is mass dependent ${ }^{2}$. The mass sensitivity of a cantilever is in the range of a few picograms ${ }^{3}$. The use of microcantilevers as force sensors in AFM is, therefore, a well-established application and takes advantage of the silicon micromachining techniques developed for integrated circuit process technology. Silicon, silicon oxide and silicon nitride cantilevers are commercially available with different shapes, dimensions and force sensitivities.

Using AFM, it is possible to directly measure streptavidin-biotin or avidin-biotin binding ${ }^{4,5}$, antigen-antibody interactions ${ }^{6}$, and hybridization of complementary DNA strand interactions ${ }^{1,7}$. In this case, functionalized silicon cantilevers carrying molecules like biotin are useful for the detection of streptavidin. Streptavidin is a molecular linker apparatus in many assays, sensors, purifications, and syntheses of importance in biotechnology. The streptavidin/biotin system is of special interest because it has one of the largest free energies of association yet observed for the non-covalent binding of a protein and small ligand in aqueous solution $\left(\mathrm{K}=10^{13} \mathrm{M}^{-1}\right)^{8}$. Streptavidin is a highly stable protein that can maintain its functional structure even under a variety of harsh conditions, such as high temperatures, etc ${ }^{9}$. These unique properties, along with the ability of biotin to be easily incorporated into various biological materials without disturbing their properties and structures, allow streptavidin to serve as a powerful, versatile tool in biomedical science and clinical diagnostics. The stability of the biotin-streptavidin complex during intensive washing permits removal of non-specifically bound and nonbiotinylated material ${ }^{10}$, this being of particular importance for the success of reaction product analysis.

One group of streptavidin applications involves solid phase assays, in which streptavidin is immobilized on a solid surface ${ }^{9,11}$. The extremely high biotin-binding affinity of streptavidin allows tight, efficient immobilization or capture of biotinylated materials on streptavidin-coated solid surfaces. The properties of this immobilized streptavidin has been studied extensively $y^{9,12}$.

Ihalainen $\mathrm{P}$ and J. Peltonen, demonstrated through Quartz Crystal Microbalance (QCM) and Scanning Probe Microscopy (SPM) measurements the successful deposition of the mixture DSDPPC (1-palmitoyl-2-(16(S-methyldithio)-hexadecanoyl)-sn-glycero-3-phosphocholine) and 1,2-dipalmitoyl-sn-glycero-3-phosphoethanolamine-N-(Cap Biotinyl) (biotinCap-DPPE) as a monolayer onto the gold substrate. This was also found to be the optimal composition for further protein immobilization studies. QCM was used to demonstrate in situ immobilization of streptavidin with a high specificity and relatively high yields onto the biotiny-lated lipid surface in liquid phase ${ }^{13}$.

E.Williams and collaborators validate the optimum conditions for the specific attachment chemistry of Silicon Carbide $(\mathrm{SiC})$ appropriate to biosensing applications (0001) $4 \mathrm{H}-\mathrm{SiC}$ was functionalized with 3 -aminopropyltriethoxisilane (APTES) and subsequently biotinylated for the selective immobilization of streptavidin. The experimental results demonstrate that the APTES functionalized and biotinylated SiC surface has the potential to be employed as a biosensing platform for the selective detection of streptavidin molecules ${ }^{14}$.

An ultrasensitive chemiluminescent (CL) immunoassay system was developed for the detection of tumor marker. This sandwich CL assay method was for the first time designed based on a highly efficient streptavidinfunctionalized Multi-walled carbon nanotubes (MWCNTs) platform. The glass slide was firstly silylanized with 3-gycidoxypropyltrimethoxysilane (GPTMS) to generate surface epoxy group functionality. Subsequently, the MWCNTs/ chitosan solution was mixed with streptavidin solution, and a certain amount of the resulting suspension was dropped on the surface of the epoxy-activated glass substrate to form a firm streptavidin-functionalized MWCNTs platform. The biofunctionalized-MWCNTs platform shows large reactive surface area and excellent biocompatibility. The capture antibody can be efficiently immobilized on the biosensing platform surface based on the highly selective recognition of streptavidin to biotinylated antibody ${ }^{15}$.

Sang-Kwon Lee reported on the rapid and direct quantification of specific cell captures using a micro-patterned streptavidin (STR)-functionalized Silicon nanowire (SiNW) platform, which was prepared by Ag-assisted wet chemical etching and a photo-lithography process. This platform operates by highaffinity cell capture rendered by the combination of antibody-epithelial cell surface-binding, biotin-streptavidin binding, and the topologically enhanced cell-substrate interaction on a 3-dimensional SiNWs array. They developed a micro-patterned nanowire platform, with which were able to directly evaluate the performance enhancement due to nanotopography. An excellent capture efficiency which is the highest value achieved thus far for the targeting specific A549 cells on a selective area of patterned SiNWs, is demonstrated ${ }^{16}$.

Moreover, to immobilize effectively oligonucleotide probes on $\mathrm{SiO}_{2}$ modified with (3-aminopropyl)triethoxysilane, four procedures based on 
streptavidin-biotin system are compared with different techniques. The first approach involves: adsorption of biotinylated Bovine Serum Albumin (BSA), blocking free surface sites with BSA, and binding of streptavidin and biotinylated oligonucleotide (b-oligo). Final steps are exchanged in the second procedure with immobilization of preformed streptavidin-b-oligo conjugate. The third approach consists of streptavidin adsorption, blocking with BSA and b-oligo binding. Finally, streptavidin-b-oligo conjugate is immobilized directly within the fourth method. All experimental methods indicate that the first and second approach, that are based on immobilization of biotinylated BSA for streptavidin attachment onto surface, lead to the more efficient streptavidin immobilization as compared to direct adsorption of streptavidin ${ }^{17}$.

The aim of this work is to explore the ability of silicon substrates to be functionalized with biotin and also to evaluate the ability of the functionalized surface to be covered by streptavidin. We have used surface analytical techniques including AFM, contact angle, and X-ray Photoelectronic Spectroscopy (XPS) to characterize the surface and to control the course of functionalization before the immobilization of streptavidin. However, such techniques do not provide information about the identity of the immobilized compounds on the surfaces. In order to confirm the presence of the fluorochrome-streptavidin on the surface we used AFM and two powerful techniques that allow us to detect and determine the identity of the immobilized streptavidin: fluorescence microscopy and MALDI-TOF, respectively. Fluorescence microscopy is a powerful technique which has been used for both the characterization of the adsorption of bacteria to a silicon surface and for the surface characterization of a siliconchip-based DNA microarray ${ }^{18}$. In this case, silicon surfaces provide a better signal-to-noise ratio than glass because silicon wafers show less background fluorescence and the dark, non-transparent surface absorbs excitation light. ${ }^{18}$ Mass spectrometry has become a principal method for the high-throughput determination of protein sequences and, more recently, to study proteinprotein and protein-ligand complexes ${ }^{19}$. The method is suitable over a large mass range, is extremely sensitive and can be applied quantitatively. The use of MALDI for the analysis of peptides adsorbed on a solid support has been reported. Moreover, the related technique Surface Enhanced Laser Desoprtion/ Ionization (SELDI) is used for the detection of different biomarkers and to study protein interactions ${ }^{20}$. The combination of laser desorption of untreated soil samples and subsequent selective laser ionisation followed by timeof-flight mass analysis results in an ultra-fast technique for the quantitative detection of aromatic contaminants in soil samples ${ }^{21}$. Furthermore, solid-phase extraction mass spectrometry methods which involve affinity capture have been developed, utilizing protein-derivatized beads affixed to the probe surface, or by using derivatized self-assembled monolayers (SAMs). For example, small amounts of peptides adsorbed on an octadecylalkyl-SAM were detected ${ }^{22}$. In our case, both techniques could be useful for the characterization of a silicon biotinylated surface with immobilized streptavidin.

In this work, we functionalized a silicon surface with biotin thorough an amino-1-alkene anchorage. We have studied the immobilization of a bioactive molecule, streptavidin, on biotin covalently bonded to a functionalized surface and show that fluorescence microscopy and MALDITOF are useful tools for the characterization of functionalized silicon surfaces, even in the case of high-molecular weight bioactive macromolecules.

\section{EXPERIMENTAL SECTION}

\section{Materials}

All chemicals were reagent grade or higher and used as received unless otherwise specified. Ultrapure water came from a Millipore system and used to rinse the surfaces to ensure clean conditions. Mesitylene (Fluka, 99\%) was distilled at atmospheric pressure and stored on $\mathrm{CaCl}_{2}$. All glassware for distillations, re-crystallizations, and monolayer preparations was cleaned with distilled solvents. Other details have been described previosly $y^{8,23,24,25}$. Si (111), n-type wafers were purchased from SILTRONIX, $5-10 \mathrm{Ohm} / \mathrm{cm}$. Streptavidin Texas red was purchased from Molecular Probes (Eugene, Oregon, USA). The conjugate has 2.9 moles dye/mole.

\section{Apparatus and procedures}

NMR: ${ }^{1} \mathrm{H}$ NMR spectra were recorded in $\mathrm{CDCl}_{3}$ at $400 \mathrm{MHz}$ on a Mercury spectrophotometer; ${ }^{13} \mathrm{C}$ NMR spectra were measured in $\mathrm{CDCl}_{3}$ at $100 \mathrm{MHz}$. Chemical shifts are in ppm relative to tetramethylsilane.

Photochemical Reactions: All photochemical reactions were performed on a Rayonet Chamber Reactor RPR-100 ultraviolet lamps $300 \mathrm{~nm}$.

Water contact angles was measured with a video camera JVC TK-1270 connected to a computer with a COMET- MATROX card using a home made program. One microliter of water was deposited on the surface. The sessile drop (which showed a spherical shape) was observed with a digital video camera. Contact angle of the sessile drop was inferred from its measured height (h) and base width or diameter (b) according to Newman and Good ${ }^{26}$. Several drops (5) were observed following the same procedure

Fluorescence Imaging. A commercial fluorescence microscope LEICA DMRB, CCCD was used for fluorescence observations. Fluorescence intensity was quantified by a Metamorph Image Analysis System v-4.0 (Universal Imaging, West Chest, PA, USA) and expressed in arbitrary units, as defined by the mean fluorescence intensity (MFI).

AFM Imaging. All AFM experiments were performed on a Digital Instruments Nanoscope III-A (Digital Instruments, Santa Barbara, CA) with standard $\mathrm{Si}_{3} \mathrm{~N}_{4}$ tips.

AFM imaging was performed in air operated in tapping mode (1-Hz scan frequency) at low tapping amplitude and at the lowest force set-point to improve resolution and minimize damage to the sample. To attain the highest resolution and least disturbance to the sample, we imaged the layers at the lowest possible oscillation amplitude, $0.1-0.2 \mathrm{~V}$ in the photodiode signal (corresponding to approximately a few nanometers RMS after amplitude calibration). For that purpose, the method that we used was to acquire simultaneously the topographic and phase signals and then reduce the oscillating amplitude (both drive and set-point), until a decrease of the topographic signal together with a sharp increase in the phase contrast was observed, possibly related with the trapping or snap-in of the tip due to capillary forces. The amplitude was then increased slightly above this value, to restore normal topography and phase at the highest resolution. Etched silicon AFM probes were used (resonant frequency $200-400 \mathrm{KHz}$, nominal spring contact $20-100 \mathrm{~N} / \mathrm{m}$, manufactured by Nanosensors).

X-ray photoelectron spectroscopy (XPS) measurements were performed in a PHI ESCA-5500.

Synthesis of 1-Chloro-10-Undecene. This compound was prepared mainly following the procedure used by Sieval et $a l^{23}$ but some steps were improved. A mixture of 10-undecylenyl alcohol, triphenylphosphine, carbon tetrachloride and acetonitril was heated to reflux for $24 \mathrm{~h}$. The resulting brown solution was cooled to room temperature and the solvents were evaporated under reduced pressure. The solid residue was transferred onto a glass filter and extracted with petroleum ether $\left(40-60^{\circ} \mathrm{C}\right)$. The combined extracts were dried over $\mathrm{MgSO}_{4}$ and concentrated under vacuum. Purification of the residue was accomplished by silica flash column chromatography (hexane/AcOEt, 9:1), and evaporation of appropriate fractions in vacuo to give $5.5 \mathrm{~g}(85 \%)$. ${ }^{1}$ H NMR: $\delta 5.90-5.69(\mathrm{~m}, 1 \mathrm{H}), 5.02-4.89(\mathrm{~m}, 2 \mathrm{H}), 3.52(\mathrm{t}, 2 \mathrm{H}, J=6.9 \mathrm{~Hz})$, 2.07-1.99 (M, 2H), 1.82-1.70 (m, 2H), 1.49-1.22(m, 12H). ${ }^{13}$ C NMR: $\delta 139.15$, $114.15,45.14,33.82,32.67,29.43,23.39,29.11,28.93,28.88,26.89$.

Synthesis of $N$-( $\omega$-Undecylenyl)-Phthalimide (I). Here also some steps were improved. $2.55 \mathrm{~g}(0.014 \mathrm{~mol})$ of potassium phthalimide was added to a solution of $2 \mathrm{~g}(0.011 \mathrm{~mol})$ of 1-chloro-10-undecene in $10 \mathrm{~mL}$ of dry DMF. The resulting suspension was stirred in an oil bath at $90^{\circ} \mathrm{C}$ for $24 \mathrm{~h}$. The resulting reaction mixture was cooled to room temperature and $30 \mathrm{~mL}$ of water was added. The aqueous layer was extracted once with $30 \mathrm{~mL}$ and subsequently twice with $12 \mathrm{~mL}$ of ether. The combined organic layers were washed with $10 \mathrm{~mL}$ of a $0.2 \mathrm{M} \mathrm{NaOH}$ solution and with $10 \mathrm{~mL}$ of brine, and dried over $\mathrm{MgSO}_{4}$. Evaporation of the solvent yielded $2.9 \mathrm{~g}(89 \%)$ of the crude product as a yellow solid. Recrystallization from $20 \mathrm{~mL}$ of distilled methanol (after standing of the solution at $18-20^{\circ} \mathrm{C}$ in a closed flask for $18 \mathrm{~h}$ ) gave $2.7 \mathrm{~g}(83 \%)$ of $N$-( $\omega$-Undecylenyl)-Phthalimide as white needles. ${ }^{1} \mathbf{H}$ NMR: $\delta$ 7.86-7.79 (m, $2 \mathrm{H}), 7.75-7.66(\mathrm{~m}, 2 \mathrm{H}), 5.90-5.72(\mathrm{~m}, 1 \mathrm{H}), 5.03-4.88(\mathrm{~m}, 2 \mathrm{H}), 6.67(\mathrm{t}, 2 \mathrm{H}, J=$ $7.3 \mathrm{~Hz}), 2.06-1.98(\mathrm{~m}, 2 \mathrm{H}), 1.71-1.58(\mathrm{~m}, 2 \mathrm{H}), 1.40-1.18(\mathrm{~m}, 12 \mathrm{H}) .{ }^{13} \mathbf{C} \mathbf{N M R}$ : $\delta 168.37,139.13,132.11,123.06,114.04,37.98,33.73,29.34,29.32,29.09$, $29.00,28.81,28.53,26.77$.

Monolayer preparation. The silicon (111) wafers, much less reactive than (100) wafers due to the absence of dangling bonds, were etched using a method similar to that described by Higashi et al ${ }^{25}$, with some modifications. A piece of silicon wafer $\left(1 \mathrm{~cm}^{2}\right)$ was placed in a teflon vial with a mixture of $\mathrm{H}_{2} \mathrm{SO}: \mathrm{H}_{2} \mathrm{O}_{2}(1: 1)$ at $80^{\circ} \mathrm{C}$ for $15 \mathrm{~min}$. Subsequently, the wafer was placed in a $40 \% \mathrm{NH}_{4} \mathrm{~F}$ solution (previously sonicated for $10 \mathrm{~min}$ after addition of several drops of ammonium sulphite $40 \%$ to avoid the presence of oxygen in the dissolution) for $20 \mathrm{~min}$ in the dark and then dried under a stream of nitrogen. The monolayers were prepared according to the following procedure. $0.2 \mathrm{M}$ of a solution of $N-(\omega$-Undecylenyl)-Phthalimide in mesitylene was placed in a small one-necked flask. The solution was deoxygenated with dry nitrogen for at least $1 \mathrm{~h}$. Subsequently, a piece of the silicon wafer (recently etched) was placed into the solution. The flask was placed in a $300 \mathrm{~nm}$ Rayonet reactor for $3 \mathrm{~h}$. The sample was then removed from the solution and cleaned in THF, methanol and dichloromethane. All samples were prepared in triplicate.

Modification of the Monolayers. Modifications with hydrazine were 
performed in closed flasks. The samples were placed in a 5\% solution (v/v) of hydrazine in distilled ethanol and the solution was stirred at room temperature for $40 \mathrm{~h}$, as described previously ${ }^{23}$. The samples were removed from the solution and cleaned in THF, methanol, and dichloromethane.

Binding of NHS-biotin to amino-terminated monolayers. Functionalized surfaces were immersed in a $0.01 \mathrm{M}$ solution of NHS-biotin in DMF under argon and allowed to react for $24 \mathrm{~h}$ room temperature. The surfaces were thoroughly rinsed with DMF, ethanol, dichloromethane and finally dried under argon.

Binding of streptavidin Texas red to biotinylated surfaces. A $60 \mu \mathrm{L}$ drop of streptavidin Texas red $(1 \mathrm{mg} / 1 \mathrm{~mL})$ was deposited on the silicon surface cover slip, the system was incubated for $1 \mathrm{~h}$ at room temperature in the dark. After the reaction, the substrates were rinsed repeatedly with large volumes of water to remove the unbound streptavidin.

MALDI-TOF. All spectra were taken in positive ion mode using a MALDI-TOF model Voyager-DE RP, equipped with a linear $337 \mathrm{~nm}$ nitrogen laser:

a)
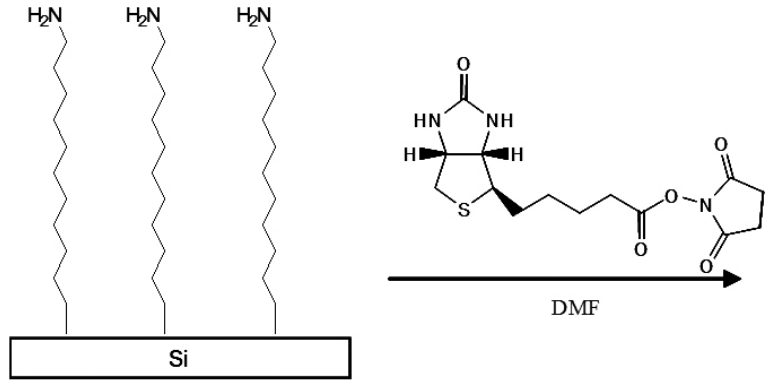

DMF

Figure 1. Scheme of a silicon functionalizated biotin amino-terminated monolayer surface. a) amino-terminated $\mathrm{H}-\mathrm{Si}$ (111) surface and b) surface obtained by treatment of amino-terminated H-Si (111) with a solution of the activated NHS-biotin molecule. b)

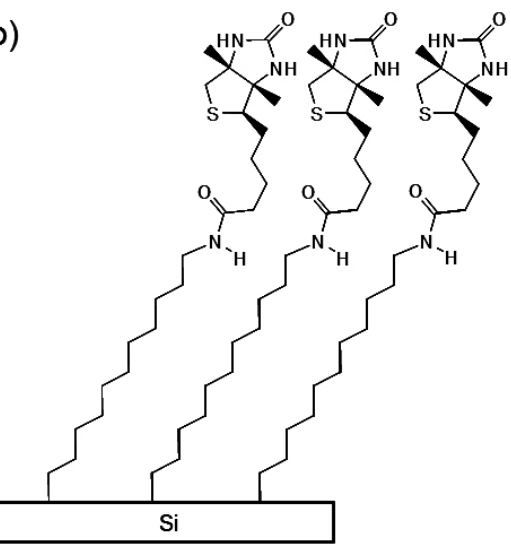

Recovery of the immobilized streptavidin from the biotinylated surface. $10 \mathrm{ml}$ of $25 \%$ ammonium hydroxide was applied to the surface and the samples were incubated for $30 \mathrm{~min}$ at $70^{\circ} \mathrm{C}$ in a wet environment. Ammonium hydroxide was then evaporated.

Mass spectrometry analysis One microliter of matrix solution (2,5-dihydroxybenzoic acid) was added to the treated surface and then the surface was dried at ambient temperature and introduced into the mass spectrometer.

\section{RESULTS AND DISCUSSION}

\section{Biotinylated monolayer on silicon}

Instead of attaching the biomolecules directly to an inorganic substrate, ultrathin self-assembled monolayers (SAMs) chemisorbed to inorganic surfaces have been used to create organic interfaces with tailor-made reactivity, structure, and properties that can be controlled by choosing the appropriate functional head group at the w-position ${ }^{27}$. In this paper we describe the attachment of biotin to an amino-terminated organic monolayer (Figure 1).
Amino-terminated monolayers have been previously used for the binding of biological material such as DNA and proteins ${ }^{23}$. In this paper a monolayer with the protected amine derivative was prepared in a convenient fashion by the reaction of I with hydrogen-terminated $\mathrm{Si}$ (111). The photochemical reaction promotes the formation of a covalently bound $\mathrm{Si}-\mathrm{C}$ between $\mathrm{N}-(\mathrm{w}-$ undecylenyl)-phtalimide (I) and Si (111). The use of amino-protecting groups is required to prevent the direct reaction of amino groups with the Si-surface, which could result in disordered monolayers.

The processing steps involving atomically flat monolayers on $\mathrm{Si}$ (111) were depicted by Wagner et $a l^{28}$. It is well established that wet chemical treatment of oxidized $\mathrm{Si}$ (111) surfaces by aqueous $\mathrm{HF}$ and $\mathrm{NH}_{4} \mathrm{~F}$ results in the termination of all surface silicon bonds by hydrogen ${ }^{25,28,29}$. An AFM image of a hydrogen terminated $\mathrm{H}-\mathrm{Si}$ (111) stepped surface is shown in figure 2. The roughness of the surface (hydrogen terminated $\mathrm{H}-\mathrm{Si}$ ) is $0.2 \mathrm{~nm}$ with the characteristic structure of atomically flat terraces.

Amino-terminated monolayers were prepared in a convenient fashion by the photochemical reaction of the protected compound I (see experimental section) with the silicon surface, followed by a deprotection reaction with hidrazine that generated the bioreactive amino-terminated monolayer. The use of amino-protecting groups is required to prevent the direct reaction of amino groups with the $\mathrm{Si}$ surface, which results in disordered monolayers ${ }^{25,26}$. An AFM image of the amino-terminated monolayer is also reported in Figure 3. surface.

Figure 2. $1 \mathrm{x} 1 \mu \mathrm{m}^{2}$ AFM image of a hydrogen-terminated H-Si (111)

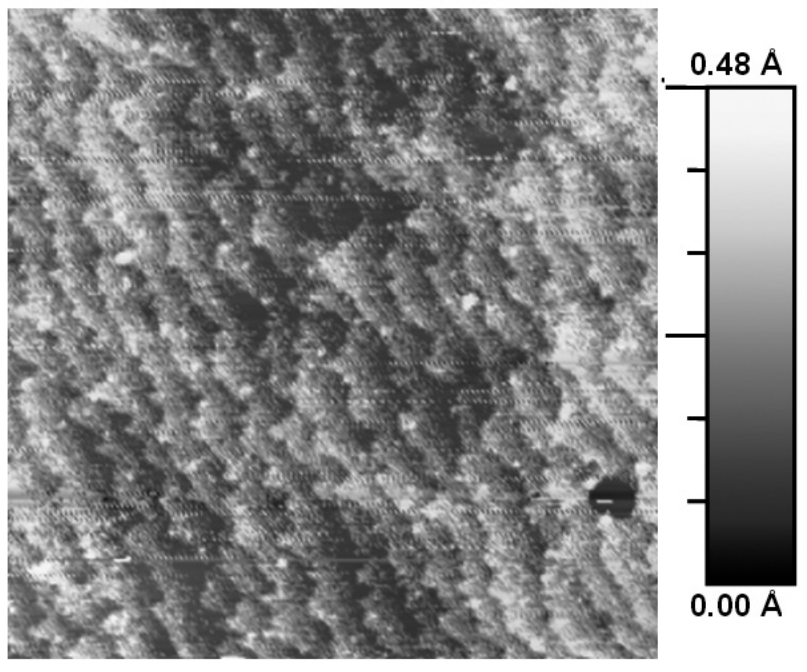




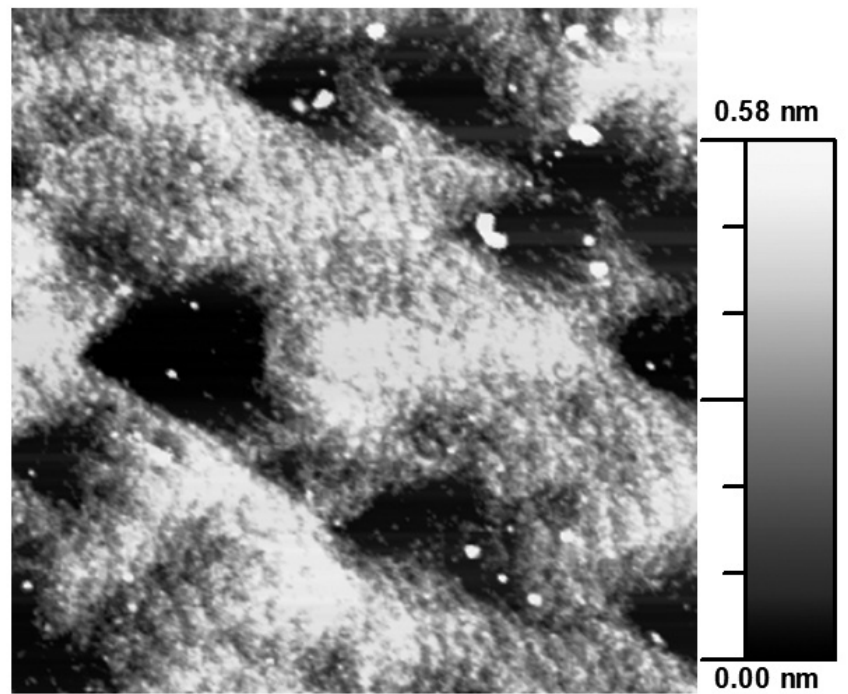

Figure 3. $1.2 \times 1.2 \mu \mathrm{m}^{2} \mathrm{AFM}$ image of an amino-terminated $\mathrm{Si}$ (111) undecyl monolayer surface.

The structural topography of the monolayer mimics that of the underlying silicon surface in the form of a stepped surface with a mean roughness of 0.25 $\mathrm{nm}$ for a similar area to that used for the non-functionalized surface. The UV light-catalyzed radical addition of alkenes does not deteriorate the flatness of the H-Si (111) surface. N-Alkylphthalimide derivatives can be converted into amines using the Ing-Manske modification of the Gabriel reaction ${ }^{23,32}$. The monolayers were reacted with a $5 \%$ solution of hydrazine in ethanol at room temperature for $48 \mathrm{~h}$. This results in a decrease in the water contact angle (a contact angle of $82^{\circ}$ for the $N$-( $\omega$-Undecylenyl)-Phthalimide functionalized surface changes to $65^{\circ}$ for the amino-terminated undecyl monolayer surface) which indicates the removal of the phthalimide groups and the emergence of the hydrophilic groups ${ }^{23,33}$. Direct detection of the amino groups in the monolayers is difficult with IR spectroscopy ${ }^{23}$ due to the formation of hydrogen bonds with adsorbed water. The monolayers were tested with XPS ${ }^{23,34,35}$ to confirm the presence of the amino groups in the monolayers. The nitrogen peak is visible at $400.6 \mathrm{eV}$ which shows that the nitrogen-containing tails of the amino-terminated compound are present in the monolayer (figure 4). The measured $\mathrm{N}(1 \mathrm{~s})$ core level binding energy is in excellent agreement with the reference value of $400.5 \mathrm{eV}$ found in imides ${ }^{27}$.

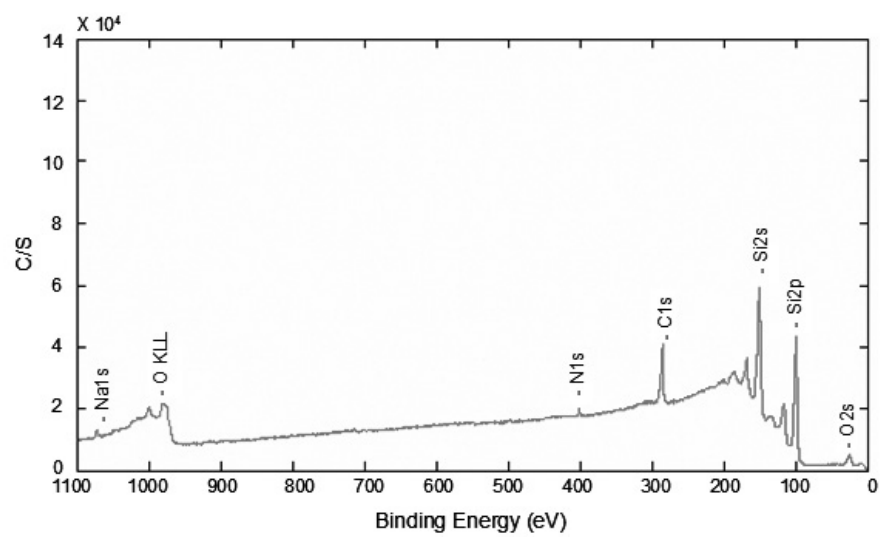

Figure 4. XPS analysis of the silicon functionalizated amino-terminated monolayer surface.

The functionalized amino-terminated monolayer ${ }^{36}$ on a silicon surface was treated with a solution of the activated NHS-biotin in DMF, as shown in the scheme of Figure 1. After being rinsed sequentially with DMF and ethanol and dried, the clean surfaces were imaged by AFM (results not shown). Features with a height of $1.4 \mathrm{~nm} \pm 0.2 \mathrm{~nm}$ were measured that are in agreement with the biotin length (1.7 $\mathrm{nm}$ in a fully extended conformation). However, AFM is not an appropriate tool for the unambiguous chemical characterization of biotin.

Immobilization of streptavidin Texas red on the biotinylated surface

Streptavidin containing the fluorochrome Texas red was immobilized on the biotinylated surface (Figure 5). The immobilization was carried out following previously reported procedures ${ }^{11,37}$. The stability of the biotinstreptavidin complex during intensive washing permits removal of nonspecifically bound and non-biotinylated material ${ }^{10}$.
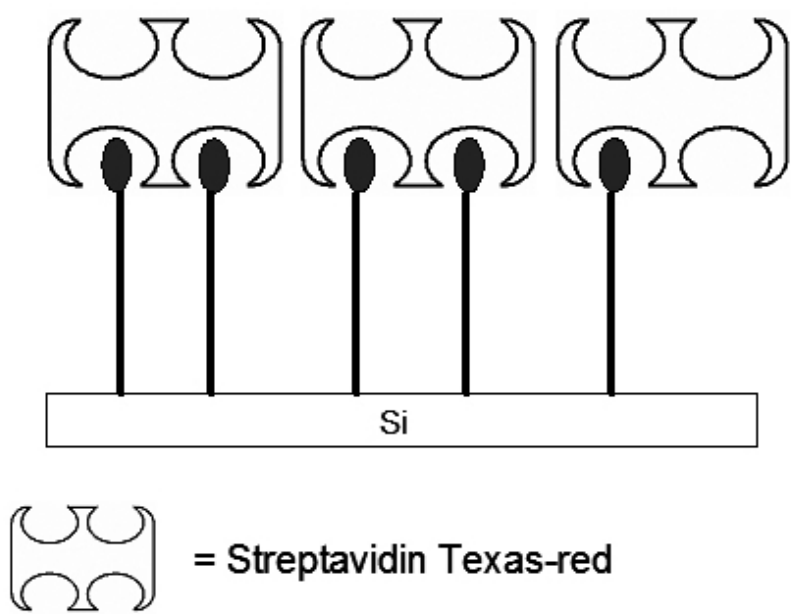

\section{$=$ Biotin}

Figure 5. Schematic representation of a streptavidin Texas red-coated surface immobilized on a biotinylated surface. In this case the streptavidin molecules interact with the biotin molecules covalently bonded to the surface through two of the four biotin binding sites and the other two sites remain free.

In order to detect the presence of streptavidin covering the biotinylated surface, fluorescence microscopy observations were performed. Figure 6 shows the presence of the Texas red chromophore covalently bonded to streptavidin immobilized on the functionalized surface. The presence of fluorescent domains distributed in all the surface is evidence of the immobilization of the streptavidin, which indicates that biotin is also present on the surface. As a negative control, we observed the amino-terminated surface (not containing biotin) treated with streptavidin, observing that there was not adsorption on the surface. The MFI values of the images of the streptavidin Texas immobilized on the biotinylated surface is four times higher than the negative control surface, the fluorescence being distributed over the entire surface, which indicates that the surface is efficiently functionalized. a)

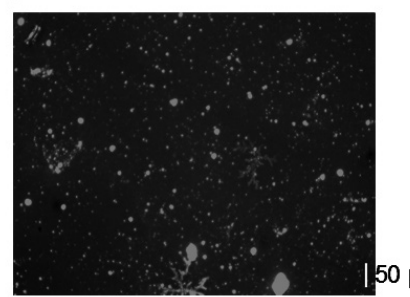

b)

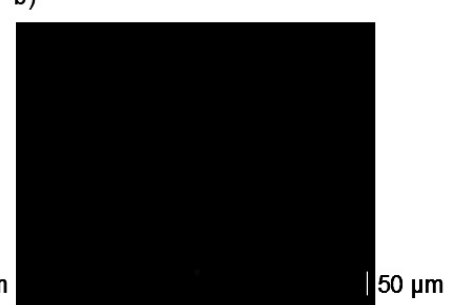

Figure 6. Fluorescence Microscopy Images: a) a biotinylated silicon surface with the immovilized streptavidin Texas red $(1 \mathrm{mg} / 1 \mathrm{~mL})$; b) Control: an amino-terminated undecyl monolayer surfaces with the immovilized streptavidin Texas red $(1 \mathrm{mg} / 1 \mathrm{~mL})$.

MFI of the biothinylated silicon surface with the immovilized streptavidin Texas red is $40 \pm 2$ and $10 \pm 2$ in control conditions. Value are the mean \pm SEM from three independent experiments performed in triplicate. MFI intensity was calculated on the entire surface images $(782 \times 582$ pixels). Images were thresholed with a value of 138 in order to avoid saturated pixels. 
The same surface was also imaged by AFM, a technique that allows $a$ priori a higher degree of resolution than fluorescence microscopy. A drop of a streptavidin aqueous solution was placed on the surface and, after being rinsed with water, the surface was imaged by AFM. Figure 7 shows the presence of aggregates of $4.5 \mathrm{~nm}$ (height) which is approximately the diameter of a streptavidin molecule ${ }^{32}$.

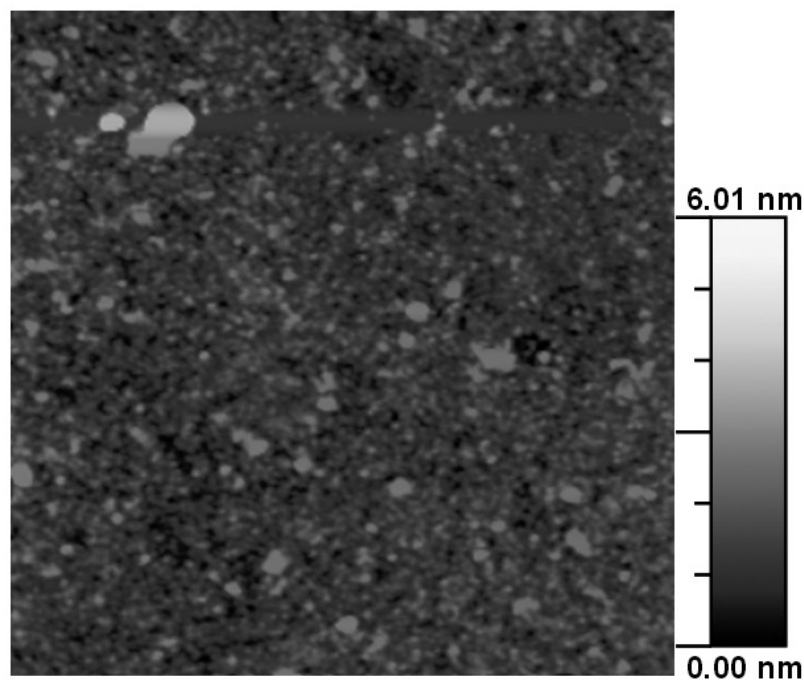

Figure 7. $2 \times 2 \mu \mathrm{m}^{2}$ AFM image of the immovilized streptavidin Texas red on the biothynilated silicon surface. A drop of a streptavidin aqueous solution was pointed on the surface and after being rinsed with water the surface was imaged.

The non-homogeneous streptavidin layer formation might occur by a crowding of the biotin alkyl molecules on the surface leading to a nonhomogeneous immobilization of the streptavidin molecules (Figure 8).

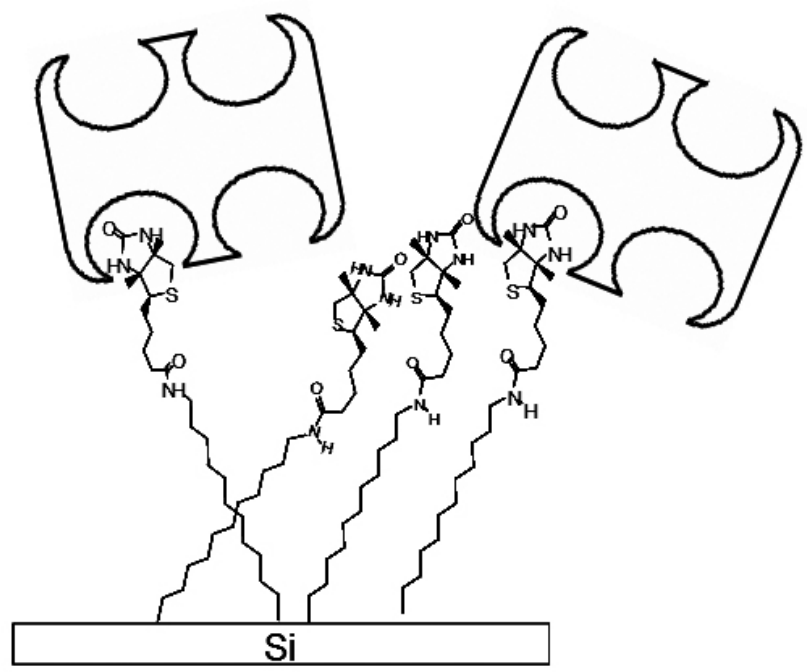

Figure 8. Scheme of the bonding structure of two molecules of streptavidin to the biotinylated monolayer on Silicon. The alkil biotinylated molecules are crowded which lead to a non homogeneous immobilization of the streptavidin molecules on the surface. The binding of two biotin sites on the same streptavidin protein to two biotins would cause molecular strain except that rare situation wherein the two surface-immobilized biotin happen to have exactly the right geometry initially to match the sites in the protein.

As proposed by Jung et $\mathrm{al}^{8}$, the crowded biotin alkyl monolayers on a surface, which produces a non-homogeneously distributed streptavidin immobilization on the surface, could be due to the differential ability of the biotin molecules to be bound to the surface. The linking of the first biotin molecule to the amino-terminated surface is relatively unrestricted (i.e., lacking steric repulsions or strains) but this first link to the surface would restrict appreciably the ability of the surface to ideally capture the second biotin molecule into the second biotin binding pocket (Figure 8). Thus, steric constrains and molecular strain probably would cause the second biotin link in the surface to be weaker, on average, than the single biotin link on a surface. Thus, binding two biotin sites on the same streptavidin protein to two rigidly immobilized biotin molecules would cause molecular strain in the protein in all cases except that rare situation wherein the two surface-immobilized biotin molecules have exactly the right initial geometry to match the sites in the protein. Surface diffusion and rotation of the biotins, if thermally allowed, may relieve some of this strain, but is unlikely to occur on a crowded surface without other energy costs 8 .

The lack of homogeneity observed in the monolayer formed by streptavidin on the silicon surface does not hamper the use of the present technology for silicon tip functionalization. As has been previously shown by other authors, tip functionalization with model organisms as bacteria, which do not form homogeneous monolayers, is very useful for AFM studies ${ }^{38}$.

In MALDI-TOF detection, streptavidin Texas red molecules immobilized on the biotinylated surface with high free energies of association were recovered from the surface after an in situ incubation at elevated temperatures and in the presence of ammonium hydroxide, leading to an efficient dissociation of biotinstreptavidin complexes as expected for the complex between streptavidin with biotinylated nucleic acids ${ }^{10}$. Figure 9 shows the MALDI spectrum of a biotinylated surface with streptavidin immobilized Texas red. The base peak is a monomer and the recorded additional peaks are for dimer, trimer, and tetramer. On the other hand, a split in both the monomer peak and in the dimer peaks was observed due to the fragmentation of the fluorochrome Texas red from the whole molecule. For the sake of comparison, the same set of samples used in fluorescence and AFM experiments were investigated by MALDI-TOF.

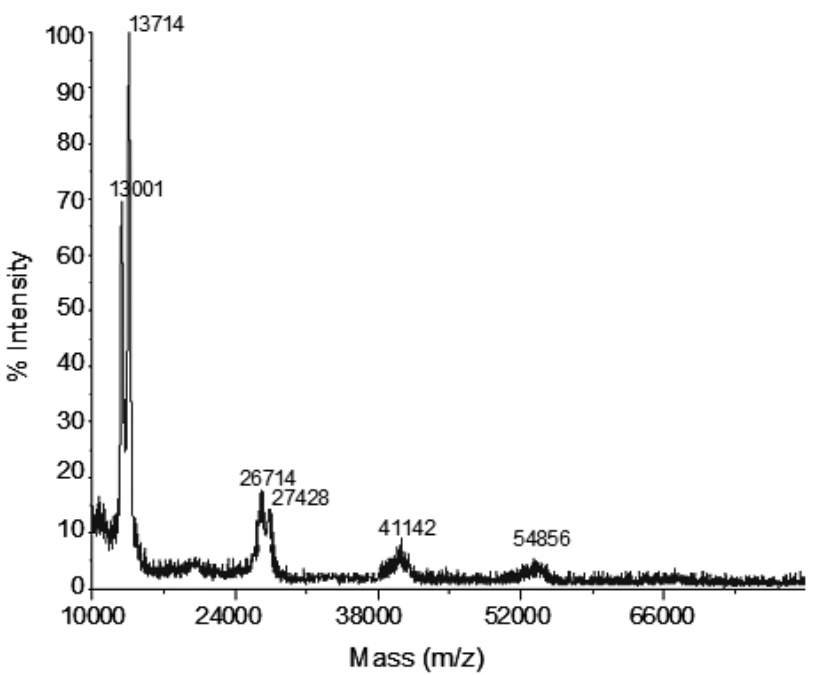

Figure 9. MALDI-TOF mass spectrum of a biotinylated surface with immobilized streptavidin Texas red. The base peak is a monomer $(\mathrm{m} / \mathrm{z}=13714)$ Additional peaks corresponding to dimer $(\mathrm{m} / \mathrm{z}=27428$, 18\% monomer intensity), trimer $(\mathrm{m} / \mathrm{z}=41142,8 \%$ monomer intensity), and tetramer $(\mathrm{m} /$ $\mathrm{z}=54856,2 \%$ monomer intensity) are also observed. A split in the monomer peak and in the dimer peaks was observed due to the fragmentation of the fluorochrome Texas red from the whole molecule.

\section{CONCLUSIONS}

We have functionalized a well defined silicon surface with biotin through a protected amino-1-alkene anchorage to the silicon external atoms. Biotin functions as a model surface with active biochemical sites for molecular detection. Streptavidin capture by the biotinylated surface has been demonstrated using two powerful techniques available in standard biochemical laboratories, fluorescence microscopy and MALDI-TOF. AFM and XPS characterization of the silicon functionalised surface can be efficient but are more difficult to implement in a sensor design laboratory and are only partial because they do not provide chemical information. 
The efficient functionalization of the surface allows it to be implemented in biochemical sensors to directly detect biomolecules. The use of silicon surfaces is rather new and functionalization can be adapted to functionalize AFM cantilevers.

\section{ACKNOWLEDGMENT}

We gratefully acknowledge financial support provided by Fondecyt 11130494, Fondap 15130011, DI-569-14/R, the Ministerio de Ciencia y Tecnología (Spain) throughout the project DPI-2000-703-C03.

\section{REFERENCES}

1. Raiteri, R.; Grattarola, M.; Butt, H. J.; Skládal, P. Sensors and Actuators B., 79, 115, (2001).

2. Davis, Z. J.; Abadal, G.; Helbo, B.; Hansen, O.; Campabadal, F.; PérezMurano, F.; Esteve, J.; Figueras, E.; Verd, J.; Barniol, N.; Boisen, A. Sensors and Actuators A., 105, 311, (2003).

3. Lange, D.; Hagleitner, C.; Hierlemann, A.; Brand, O.; Baltes, H. Anal. Chem. 74, 3084, (2002).

4. Florin, E. L.; Moy, V. T.; Gaub, H. E. Science. 264, 415, (1994).

5. Lee, G. U.; Kidwell, D. A.; Colton, R. J. Langmuir., 10, 354, (1994).

6. Browning-Kelley, M. E.; Wadu-Mesthrige, K.; Hari, V.; Chen, G. Y. Langmuir., 13, 343, (1997).

7. Lee, G. U.; Chrisey, L. A.; Colton, R. J. Science. 266, 771, (1994).

8. Jung, L. S.; Nelson, K. E.; Stayton, P. S.; Campbell, C. T. Langmuir. 16, 9421, (2000).

9. Reznik, G. O.; Vajda, S.; Cantor, C. R.; Sano, T. Bioconjugate Chem. 12, $1000,(2001)$.

10. Jurinke, C.; van den Boom, D.; Collazo, V.; Lüchow, A.; Jacob.A; Köster, H. Anal.Chem., 69, 904, (1997).

11. Berquand, A.; Mazeran, P. E.; Pantigny, J.; Proux-Delrouyre, V.; aval, J. M.; ourdillon, C. Langmuir. 19, 1700, (2003).

12. Pradier, C. M.; Salmain, M.; Zheng, L.; Jaouen, G. Surface Science. 502, 193, (2002)

13. Ihalainen P.; Peltonen J. Sensors and actuators B 102, 207, (2004).

14. Williams, E.; Pavydov A.; Motayed A.; Sundaresan S.; Bocchini P.; Richter L.; Stan G.; Steffens K.; Zangmeister R.; Schreifels J.; Mulpuriv R. Applied surface science 258, 6056, (2012).

15. Yang Z.; Shen J.; Zhu J.; Hu X. Analytica Chimica Acta, 774, 85 (2013).

16. Sang-Kovon L.; Dong-Joo K.; GeeHee L.; Gil-Sung K.; Minsuk Kuck, R. Biosensors and Bioelectronics, 54, 181, (2014).
17. Awsiulk K.; Rysz J.; Petrou P.; Bidkowski B.; Bernasik A.; Kakabakos S.; Marzec, M.; Rapter I. Applied surface science, 290, 199, (2014).

18. Lenigk, R.; Carles, M.; Ip, N. Y.; Sucher, N. J. Langmuir. 17, 2497, (2001).

19. Villanueva, J.; Yanes, O.; Querol, E.; Serrano, L.; Aviles, F. X. Anal. Chem. 75, 3385 (2003).

20. Van Bon L.; Cossu M.; Loof A.; Gohar F.; Wittkowski H.; Vonk M.; Roth J.; van den Berg W.; van Heerde W.; Broen JCA.; Radstake TRDJ. Ann Rheum Dis 2014; 0:1-5. doi:10.1136/annrheumdis-2013-205013

21. Weickhardt, C.; Tönnies, K.; Globig, D Anal.Chem. 74, 4861, (2002).

22. Brockman, A. H.; Shah, N. N.; Orlando, R. Journal of Mass Spectrometry. 33, 1141, (1998).

23. Sieval, A. B.; Linke, R.; Heij, G.; Meijer, G.; Zuilhof, H.; Sudhölter, E. J. R. Langmuir. 17, 7554, (2001).

24. Sieval, A. B.; Vleeming, V.; Zuilhof, N.; Dhölter, E. J. R. Langmuir., 15, 8288, (1999).

25. Higashi, G. S.; Chabal, Y. J.; Trucks, G. W.; Raghavachari, K. Appl. Phys. Lett., 56, 656, (1990).

26. Newman, A. W.; Good R.J; Techniques of measuring contact angle. In Surface and Colloid Sciences: Experimental Methods, Good R.J.; Stromberg R.R., Eds.; Plenum Press: New York ed.; 33, (1979).

27. Ulman, A. Chem.Rev. 96, 1533 (1996).

28. Wagner, P.; Nock, S.; Spudich, J. A.; Volkmuth, W. D.; Chu, S.; Cicero, R. L.; Wade, C. P.; Linford, M. R.; Chidsey, C. E. D. J. Struct.Biol., 119, $189,(1997)$

29. Buriak, J. M. Chemical Reviews. 102, 1271, (2002).

30. Bitzer, T.; Alkunshalie, T.; Richardson, N. V. Surf. Sci. 368, 202, (1996)

31. Zhu, X. Y.; Mulder, J. A.; Bergerson, W. F. Langmuir. 15, 8147, (1999)

32. March, J. J. Advanced Organic Chemistry. 4th ed, 425, (1992)

33. Heise, A. M.; Yim, H.; Foster, M. D.; Wieringa, R. H.; Schouten, A. J.; Erb, V.; Stamm, M. Langmuir. 13, 723, (1997).

34. Sieval, A. B.; Linke, R.; Zuihof, H.; Sudhölter, E. J. R. Adv. Mater. 12, 1457, (2000).

35. Linford, M. R; Foster, M. D; Eisenberger, P. M; Chidsey, C. E. D J. Am. Chem. Soc. 117, 3145, (1995).

36. Yam, C. M.; Pradier, C. M.; Salmain, M.; Marcus, P.; Jaouen, G. Journal of Colloid and Interface Science. 235, 183, (2001)

37. Wong, J.; Chilkoti, A.; Moy, V. T Biomolecular Engineering. 16, 45, (1999).

38. Ong, Y. L.; Razatos, A.; Georgiou, G.; Sharma, M. M. Langmuir. 15, 2719, (1999). 